\title{
ESTUDO DE VARIABILIDADE ESTELAR EM ESTRELAS SUB ANÃS QUENTES USANDO A TÉCNICA DE ANÁLISE DE FLUTUAÇÕES DEPURADAS DE TENDÊNCIAS
}

\author{
Bianca Ramielly Bomfim de Jesus ${ }^{1}$; Marildo Geraldête Pereira ${ }^{2}$ \\ 1. Bolsista FAPESB, Graduanda em Bacharelado em Física, Universidade Estadual de Feira de Santana, e-mail: \\ biancaramielly@hotmail.com \\ 2. Orientador, Departamento de Física, Universidade Estadual de Feira de Santana, e-mail: marildogp@gmail.com
}

PALAVRAS-CHAVE: Fotometria; Variabilidade Estelar; $D F A$.

\section{INTRODUÇÃO}

A Variabilidade estelar é uma das mais populares e dinâmicas áreas da pesquisa astronômica moderna, pois esta é uma propriedade que está presente na maioria das estrelas, e como tal tem muito a contribuir com a nossa compreensão das mesmas. Estrelas variáveis nos provêm com parâmetros adicionais (escalas temporais, amplitudes,...), os quais são disponíveis apenas para estrelas desse tipo. Estes parâmetros podem ser utilizados para deduzir propriedades físicas, como massa, raio, luminosidade, rotação, etc. Na astronomia observacional, os fenômenos de variabilidade podem ser observados através da fotometria da radiação eletromagnética proveniente da estrela, a qual é transformada em uma série temporal, a qual descreve como varia o brilho da fonte em função do tempo. Estas séries temporais, chamadas de curvas de luz, podem ser analisadas por diversos métodos, isto vai depender exclusivamente do objeto de interesse.

Uma classe de estrelas que tem despertado interesse são as estrelas sub anãs quentes, de tipo espectral $\mathrm{O}$ e B. Essas estrelas representam os estágios finais da vida de estrelas de baixa massa. Sendo encontradas entre a sequência principal e o ramo das anãs brancas no diagrama Hertzprung-Russel (HR).

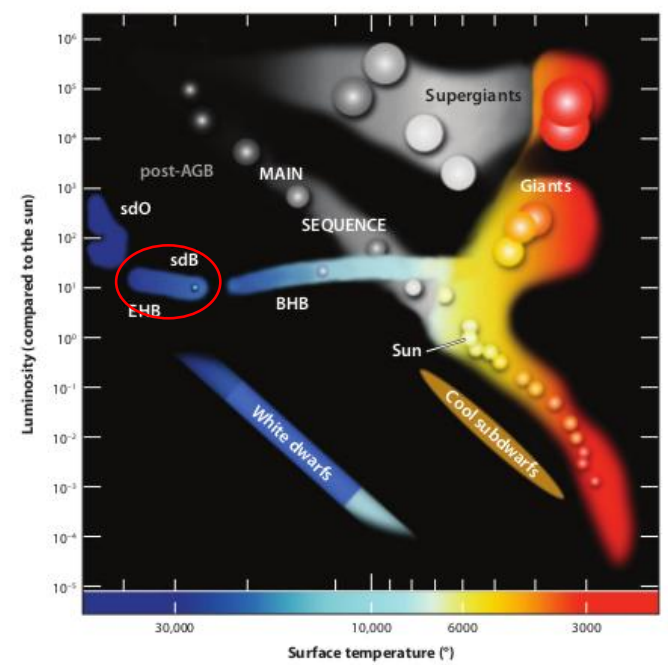

Figura 1. Diagrama HR com apresentação das estrelas Sub anãs no Ramo Horizontal Extremo. Fonte: Heber, 2009

A primeira estrela pulsante sdB descoberta foi EC 14026 e acabou tendo o nome de estrelas variáveis do tipo V361 Hya (Kilkenny et al., 1997). Estas estrelas apresentamse como pulsadores multimodais com períodos típicos da ordem de 80-600s. A origem teórica destes objetos foi prevista por (Charpinet et al., 1996). Posteriormente, foram 
encontradas estrelas deste tipo com períodos de longa duração e outras com propriedades híbridas (Krzesinski et al., 2014).

Lançado em 2009 pela NASA, a missão primária do satélite Kepler era identificar planetas equivalentes a terra pelo método de trânsito. Porém algumas estrelas da classe sdB (Sub Anãs de Tipo Espectral B) acabaram sendo encontradas no campo de observação do telescópio. Estas estrelas acabaram tendo especial atenção devido à raridade de se encontra-las e foram observadas no modo de curta cadência, o que se apresenta como uma grande oportunidade de se observar estes objetos com boa resolução temporal em uma escala de tempo muito longa. Estes dois aspectos observacionais juntos permitem avaliar como as pulsações variam com o tempo.

Os dados públicos do Kepler podem ser acessados no NASA exoplanet archive ${ }^{l}$. Os dados podem ser baixados em Longa Cadência (obtém uma integração a cada $30 \mathrm{~min}$ ) ou no nosso caso, Curta Cadência (obtém uma integração a cada 58.85s).

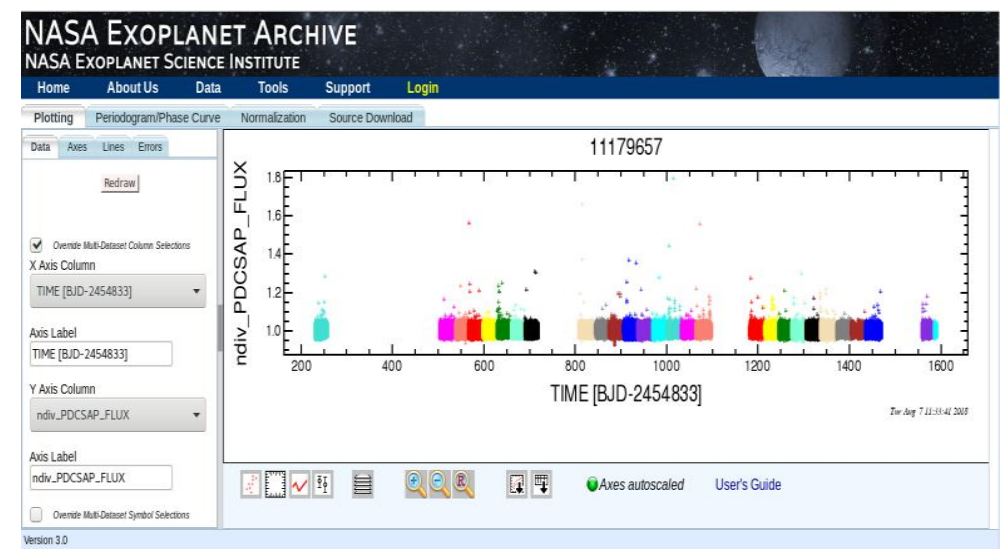

Figura 2. Exemplo de uma curva de luz normalizada por divisão no banco de dados do Kepler.

\section{MATERIAL E MÉTODOS OU METODOLOGIA (ou equivalente)}

Para a análise das curvas de luz, utilizamos o método de Análise de Flutuações Depuradas de Tendências (do inglês Detrended Fluctuation Analisys - DFA).

O método DFA foi desenvolvido em (Peng et al., 1994), e consiste em se determinar a memória de escala (ou correlação de longo alcance) de um determinado fenômeno. Aqui utilizaremos o DFA para analisar a correlação de longo alcance nas curvas de luz de estrelas tipo sdB presentes nos dados públicos do satélite Kepler. Análises realizadas em trabalhos anteriores tem indicado um comportamento nas curvas de luz com mais de um coeficiente de correlação. Para cada escala temporal relacionada com um coeficiente de correlação são determinados os limites temporais de validade para aquele dado coeficiente.

Ao aplicar o método $\mathrm{DFA}^{2}$ às curvas de luz, o programa gera um gráfico, conforme observado na Fig. 3. Após isto, para cada escala temporal relacionada com um coeficiente de correlação $\alpha$ são determinados os limites temporais de variabilidade para aquele dado coeficiente.

1 http : //exoplanetarchive.ipac.caltech.edu

${ }^{2}$ O código DFA pode ser encontrado em: https://www.physionet.org/physiotools/dfa/ 


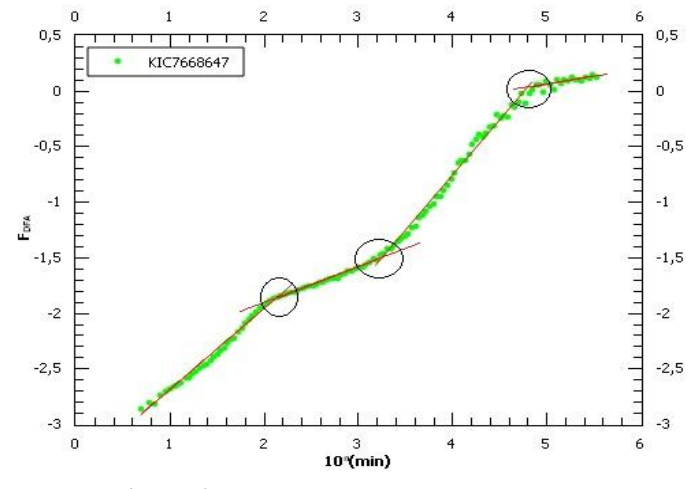

Figura 3. Curva de luz após o uso do DFA

No gráfico contido na fig.3, podemos encontrar o $\alpha_{\mathrm{DFA}}$ a partir da regressão linear das partes lineares entre as intersecções. A intersecção das linhas contínuas em $\mathrm{F}_{\mathrm{DFA}}(\mathrm{n})$ representa o limite da transição de um tipo de variabilidade para outra, e o valor do expoente $\alpha_{\mathrm{DFA}}$ comprova este fato (Zebende et al 2016).

\section{RESULTADOS E/OU DISCUSSÃO (ou Análise e discussão dos resultados)}

Através da investigação sobre fontes do Kepler na literatura; Ostensen et al.(2011); Ostensen et al.(2010), podemos construir uma tabela com 15 fontes sdBs.

Aplicando o DFA nessas fontes podemos perceber que 54\% das fontes apresentam quatro escalas de correlação, $40 \%$ apresentam três escalas e apenas $6 \%$ apresentam cinco regimes de $\alpha$. Para obtermos uma melhor compreensão sobre o comportamento dos coeficientes de correlação, fizemos um gráfico da variação dos $\alpha$ com relação ao tempo.

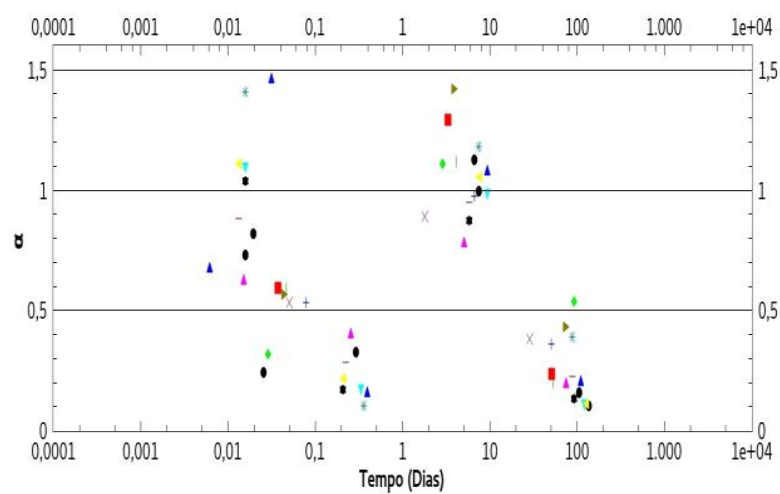

Figura 4. Gráfico mostrando o comportamento de $\alpha$ como função das escalas de tempo de variabilidade das fontes.

Ao analisarmos o gráfico do comportamento de $\alpha$ com relação ao tempo (FIG. 4), podese perceber a divisão do sinal temporal em regiões distintas. Os valores obtidos de $\alpha$ para cada escala de tempo é interpretado em termos das características das variações de brilho em diferentes escalas, desta forma depreendemos do observado na FIG. 4 e obtemos que para escalas de tempo entre 0,005 e 0,01 dias as séries apresentam uma persistência de longo alcance. Para escalas entre 0,1 e 1 dias e 20 e 200 dias as séries apresentam um comportamento antipersistente, que indica uma progressiva perda de memória e para escalas de tempo ente 1 e 10 dias as séries apresentam um comportamento de ruído rosa ou browniano.

\section{Conclusão}

Com esse trabalho tínhamos o objetivo de estudar as escalas de tempo de correlação de longo alcance em um grupo de estrelas sdB usando o método DFA. Nesse sentido foram analisadas 15 fontes sdBs isoladas, observadas pelo satélite Kepler. 
As curvas de luz ao serem analisadas com a técnica DFA mostraram não ser possível a descrição total de uma determinada fonte em termos de uma única lei de potência, com exceção das fontes KIC 4244427, KIC 7664467 e KIC830219 as quais apresentam sutis variações da lei de potencia. Já as outras 12 fontes apresentam um caráter onde há a necessidade de mais de uma lei de potencia, com indícios de características similares a multifractalidade, como evidenciado em alguns outros problemas não astrofísicos (Kantelhardt et al, 2002). Este tipo de análise também foi feito para o estudo de manchas solares (Mohaved et al, 2006).

Analisando de forma individual cada componente presente nas curvas de correlação $D F A$, pode-se medir cada um dos coeficientes de correlação de longo alcance. A análise desses coeficientes mostrou um comportamento persistente na região de alta frequência, com $\alpha_{1}$ variando entre 0,5 e 1 , com escala de tempo da ordem de minuto/horas. A banda relacionada aos coeficientes $\alpha_{2}$, apresentam um comportamento antipersistente com alguns casos dando indício de total perda de memória de escala com coeficiente $\alpha$ se aproximando de 0 . Já nas regiões de baixa frequência com escalas de tempo entre 1 e 10 dias observa-se que os alfas apresentam valores superiores a 0,75 chegando a 1,5 , esse comportamento é característico as flutuações de brilho observadas em sinais semelhantes ao ruído vermelho. Nas escalas de tempo da ordem de 100 dias novamente o sinal tende a uma perda de correlação.

\section{REFERÊNCIAS}

KILKENNY, D. et al. A new class of rapidly pulsating star-I. EC 14026-2647, the class prototype. Monthly Notices of the Royal Astronomical Society, v. 285, n. 3, p. 640-644, 1997.

KRZESINSKI, J. et al. Constraining the Hydrogen Envelope Mass of a Pulsating sdB Star KIC 10670103 Through Asteroseismology. Acta Astronomica, v. 64, n. 2, p. 151165, 2014.

PENG, C.-K. et al. Mosaic organization of DNA nucleotides. Physical review e, v. 49, n. 2, p. 1685, 1994.

CHARPINET, S. et al. The potential of asteroseismology for hot, subdwarf B stars: a new class of pulsating stars?. The Astrophysical Journal Letters, v. 471, n. 2, p. L103, 1996.

ZEBENDE, G. F. et al. Analysis of the variability in the sdB star KIC 10670103: DFA approach. Monthly Notices of the Royal Astronomical Society, v. 464, n. 3, p. 2638 2642, 2016.

ØSTENSEN, Roy H. et al. First Kepler results on compact pulsators-VI. Targets in the final half of the survey phase. Monthly Notices of the Royal Astronomical Society, v. 414, n. 4, p. 2860-2870, 2011.

ØSTENSEN, Roy H. et al. First Kepler results on compact pulsators-I. Survey target selection and the first pulsators. Monthly Notices of the Royal Astronomical Society, v. 409, n. 4, p. 1470-1486, 2010.

CHEN, Zhi et al. Effect of nonstationarities on detrended fluctuation analysis. Physical review E, v. 65, n. 4, p. 041107, 2002.

KANTELHARDT, Jan W. et al. Multifractal detrended fluctuation analysis of nonstationary time series. Physica A: Statistical Mechanics and its Applications, v. 316, n. 1-4, p. 87-114, 2002.

MOVAHED, M. Sadegh et al. Multifractal detrended fluctuation analysis of sunspot time series. Journal of Statistical Mechanics: Theory and Experiment, v. 2006, n. 02, p. P02003, 2006. 